\title{
Switching States: dynamic remodelling of polarity complexes as a toolkit for cell polarization
}

Florent Peglion ${ }^{1}$ and Nathan W. Goehring ${ }^{2,3}$

${ }^{1}$ Cell Polarity, Migration and Cancer Unit, Institut Pasteur, UMR3691 CNRS, Equipe Labellisée Ligue Contre le Cancer, F-75015, Paris, France.

2The Francis Crick Institute, London, UK

${ }^{3}$ MRC Laboratory for Molecular Cell Biology, UCL, London, UK

\begin{abstract}
Polarity is defined by the segregation of cellular components along a defined axis. To polarize robustly, cells must be able to break symmetry and subsequently amplify these nascent asymmetries. Finally, asymmetric localization of signaling molecules must be translated into functional regulation of downstream effector pathways. Central to these behaviors are a diverse set of cell polarity networks. Within these networks, molecules exhibit varied behaviors, dynamically switching among different complexes and states, active vs inactive, bound vs unbound, immobile vs diffusive. This ability to switch dynamically between states is intimately connected to the ability of molecules to generate asymmetric patterns within cells. Focusing primarily on polarity pathways governed by the conserved PAR proteins, we discuss strategies enabled by these dynamic behaviors that are used by cells to polarize. We highlight not only how switching between states is linked to the ability of polarity proteins to localize asymmetrically, but also how cells take advantage of 'state switching' to regulate polarity in time and space.
\end{abstract}

Word count: 2870 


\section{Introduction}

Cell polarity describes the functional asymmetry of cells along a defined axis. Polarized cells typically possess complex molecular networks that orchestrate the asymmetric segregation of molecules, classically in response to local cues, and translate these molecular asymmetries to spatially control downstream effector pathways. Polarity networks are incredibly diverse, with distinct pathways operating not only in different species, but also within different cells of an organism or even at different times in the same cell. Although we will focus on the polarity of animal cells, and in particular, pathways related to the conserved PAR cell polarity network, we hope to illustrate principles common to the diversity of cell polarity networks and the cells in which they operate.

Animal cell polarity is typically associated with conserved networks of polarity regulators. One of the most widespread is the par-titioning defective or PAR proteins pathway, which includes the scaffolds Par3 and Par6, the atypical protein kinase C (aPKC) and the small GTPase Cdc42. This PAR complex is typically opposed by lethal giant larva (Lgl) and Par1 (Mark) kinase, along with context-specific components such as PAR-2 in C. elegans, or SImb (SCF E3 ubiquitin ligase) and Lulu/Yurt in higher animals [1-5]. These core PAR proteins are associated with a variety of other players. In epithelia, aPKC associates with the apical Crumbs complex - Crumbs, Stardust (Pals1) and PatJ , while basolateral membranes are defined by Scribble and Discs-Large (Dlg), in concert with Lgl and Par1 $[6,7]$. Together these molecules are involved in both the integration of geometric cues and the regulation of numerous downstream processes to functionally polarize cells. Work over the past decades has revealed that far from existing as stable complexes with a single function, these molecules are engaged in dynamic changes in interactions, localization patterns, and even molecular function [8].

This mini-review will focus on the dynamic nature of these polarity complexes and related molecules in the pathways they regulate. Specifically, we focus on their capacity to switch between states and how such switching enables polarity to be both robust and adaptable to the diversity of contexts in which these molecules act. Whether this switching is between active and inactive forms, bound and unbound, clustered and non-clustered, condensed and soluble, it is increasingly clear that such switching events control the ability of molecules to segregate in space and enable their function to be regulated in time and space. Ultimately, it is this adaptability of these networks that has enabled their use across a broad range of morphological events, including, but not limited to asymmetric cell division and fate specification, establishment of cell and tissue architecture and directional cell migration. 


\section{Pathways to Asymmetry}

Entropy dictates that the random motion of molecules will tend to dissipate asymmetries in a system. Therefore, the generation of polarized distributions of molecules across the cell requires mechanisms to induce and amplify local concentration differences. Cells have consequently developed active processes for inducing asymmetry, including spatial varying production/degradation (source-sink), biased transport or local retention. Here we focus on the latter two mechanisms in which state switching allows for local accumulation.

\section{Active and Passive Directed Transport}

An intuitively simple mechanism to drive asymmetry is through active and directed transport of polarity molecules. This is typically accomplished by molecular motors running on cytoskeletal networks, with directional transport arising from bias in the orientation of cytoskeletal tracks and association of cargo with motors that move preferentially in one direction along them. A classic example is transport of oskar mRNA by Kinesin 1 in the Drosophila oocyte. Here, the plus-end directed motion of kinesin, combined with a weak PAR-1-dependent bias in microtubule orientation, drives oskar accumulation at the posterior of oocyte[9,10] (Figure 1A). Directional motor-driven transport similarly underlies transport of Cdc42- and Rac-enriched vacuoles to the nascent apical surface of endothelial cells in support of tubule formation[11,12], and polarized transport of PAR-3 to the apical and junctional membranes in endothelia and epithelia[13], and to axon growth cones in neural cells[14].

In addition to asymmetric transport networks, polarized transport requires regulation of cargo binding and switching between transport competent and incompetent states. For Par3, a switch in motor preferences allows for cell-type specific orientation of Par3 polarity relative to the underlying polarity of the microtubule network. A preference for the microtubule minus enddirected motor dynein allows Par3 to reach polarized junctions during epithelial cellularisation, junctions in collectively migrating fibroblasts, and the anterior of the Drosophila oocyte $[13,15,16]$ . By contrast, a switch to the plus end-directed kinesin motor KIF3A allows Par3 to segregate to the tip of future axons in neurons [14]. PAR proteins in turn can regulate asymmetric transport. In migratory cells, leading edge enrichment of PAR proteins switches the relative forward (kinesin) and rearward (dynein) flux of intermediate filaments (IF) to promote transport towards the leading edge [17] (Figure 1B). 
Directed transport can also arise indirectly through bulk flows, independently of direct motordriven transport. For example, in lamellipodia of migrating cells, preferential nucleation and polymerization of actin at the cell front is coupled to contractile activity of myosin at the rear to drive a rearward bulk flow of actin filaments in a thin layer under the membrane known as the cortex [18-20]. Retrograde flow can induce gradients of a broad range of molecules associated with actin or even simply entangled or embedded within this viscous layer, to sustain long-lasting cell polarization of migrating cell [21]. Ultimately, the magnitude of polarity for a given species was shown to be directly related to the timescale of switching between actin-associated and free states, revealing how bias in switching rates can help shape distributions of molecules in such systems [13] (Figure 1C).

Such flow-dependent transport drives polarization of $C$. elegans zygote. Here, PAR-3/PAR6/PKC-3(aPKC) are actively segregated into the anterior, not through direct association with motors, but by anterior-directed flows of the membrane-associated actomyosin cortex [22,23]. A recent trio of studies revealed PAR-6 and PKC-3 switch between alternate PAR-3- and CDC-42associated states. Cortical flow-dependent transport or 'advection' of these molecules by flow depends specifically on the PAR-3 state, which is characterized by long-lived, slowly diffusing clusters, that are carried by the flow and actively promoted during polarization [24-26] (Figure 1C).

\section{Local Retention}

State switching also plays a key role in generating cell polarity through diffusion-based retention mechanisms, which require the interconversion of molecules between slow- and fast-moving states. Spatial regulation of the rates at which molecules shift between these two states biases the effective diffusion of molecules across the cell, and provides a mechanism for asymmetric accumulation (Figure 2).

One common paradigm that illustrates this concept is the case of switching between inactive, freely diffusing cytoplasmic states and an active, slowly diffusing membrane states. Because of their reduced diffusion, molecules loaded onto the membrane in one location will not immediately diffuse away, allowing them to accumulate locally. This local accumulation creates an effective sink for cytoplasmic molecules as they are recruited to the membrane, causing net diffusion of cytoplasmic molecules from elsewhere in the cell to balance local binding events. This local 
accumulation, which may be driven by upregulation of membrane binding or reduction in dissociation, when coupled to diffusive transport from elsewhere in the cell, gives rise to a polarized distribution.

In the $C$. elegans zygote, PAR proteins ultimately segregate into two opposing membrane domains. Fluorescence recovery after photobleaching (FRAP) provided the first measures of membrane diffusion and dissociation in the embryo, supporting a model in which spatial variation in membrane binding allowed accumulation of slower diffusing species within domains drawn from a rapidly diffusing cytoplasmic pool [27]. Recent single molecule methods have provided further insight into the spatially biased turnover of PAR proteins, suggesting that while posterior proteins primarily bias membrane association of anterior proteins, most likely through limiting availability of membrane-binding adapter molecules PAR-3 and Cdc42 [28], posterior protein distributions are driven primarily by an anterior bias in dissociation downstream of the kinase PKC-3 [29,30] .

In such models, understanding the mechanisms of membrane association and how it restricts diffusion becomes paramount. Regulated binding of plasma membrane lipids has emerged as a common theme. The polarity-related proteins Par1, Lgl, Miranda, Numb, PAR-2 and the anterior protein Par3 share a common ability to bind anionic phospholipids, most likely the plasma membrane-enriched PIP2, via dedicated domains [31-36]. A shared characteristic of numerous polarity-related proteins, these domains are often the direct targets of regulatory kinases, allowing membrane association to be regulated in time and space. Miranda, Numb, Lgl and PAR-2 membrane binding domains are direct targets of the polarity-related kinase aPKC, providing a mechanism for exclusion from aPKC-enriched domains [32,34,36,37].

The affinity of single lipid binding domains is often insufficient to allow stable membrane association and restriction of diffusion. Instead, molecules typically engage in multivalent association with the membrane through the formation of homo- or hetero-dimeric complexes (Figure 2A). PAR-2 and Par3 form homo-oligomers [30,38], while PAR-1 and the related MARK kinases are thought to require coincident binding to plasma membrane lipids along with additional accessory factors, such as the $C$. elegans PAR-2 protein, to achieve maximal membrane enrichment [31]. In the case of Par3, it is precisely these higher-order, multivalent interactions that allow formation of stable, slow-diffusing, membrane-associated clusters that are segregated by cortical flows as discussed above and which likely play a role in numerous systems $[38,39]$. 
Assembly of complexes then becomes an additional point for regulating switching between fast cytoplasmic and slow membrane-associated states, with aPKC proposed to disfavor PAR-2 oligomerization [30] and PAR-1/PAR-2 heterodimer formation [31], and PAR-1 impeding PAR-3 clusters [28].

While membrane association is a common paradigm for regulating mobility in polarity networks in bacteria, yeast, plants, and animals, mechanisms for inducing spatial variation in diffusivity are much broader. One such case is the formation of asymmetries of cytoplasmic proteins, the most well-studied examples occurring the $C$. elegans zygote. Here, during the first cell division, asymmetries of various fate determinants are set up along the anterior-posterior axis to induce cell fate differences in the two daughters. Despite not ostensively requiring membrane binding, the general paradigm of regulated switching between fast and slow diffusing states is similar, with slow diffusing species accumulating preferentially on one size of the cell. The key difference is in how the slow diffusing state is achieved.

One way to restrict mobility is through binding of cytoplasmic RNA (Figure 2B). mRNAs diffuse very slowly in eukaryotic cytoplasm $\left(<0.4 \mu \mathrm{m}^{2} / \mathrm{s}\right.$ [40,41] vs $>20 \mu \mathrm{m}^{2} / \mathrm{s}$ for soluble GFP [42]) and thus reversible RNA binding would allow for local switching of mobility between slow, RNAassociated states and fast, unbound states. In the zygote, biases in mobility were first observed for two cytoplasmic fate determinants downstream of PAR polarity, MEX-5 and PIE-1 [43,44]. Polarity of MEX-5 was subsequently shown to require RNA-binding, with the switch between bound and unbound states regulated by the opposed activity of the polarized kinase PAR-1, which promotes the unbound, fast state, and a uniform phosphatase PP2A that catalysed the reverse [45]. Quantitative imaging and single particle tracking have now extended this paradigm of RNAbinding and spatial variation in switching rates to PIE-1, POS-1, and MEG-3 [46-49].

Liquid-liquid phase separation (LLPS) has emerged as another common theme for structuring the cytoplasm, allowing the local enrichment or sequestration of molecules within membraneless compartments. Liquid droplets diffuse at extremely slow rates. Thus, when a soluble molecule partitions into the liquid droplet phase, they are effectively immobilized, allowing them to accumulate locally. LLPS governs asymmetric segregation of germ granules in a number of systems (Figure 2C). In the $C$. elegans zygote, LLPS is critical for asymmetry of the germline associated $\mathrm{P}$ granules, which are large phase separated droplets composed of diverse proteins 
and mRNAs [50]. Here LLPS is controlled by MEX-5. Anterior-enriched MEX-5 locally suppresses phase separation through competition with $\mathrm{P}$ granule components for RNA binding sites, ultimately restricting $\mathrm{P}$ granules to the posterior $[51,52]$. LLPS has been proposed to play a role in numerous polarity pathways, including in the segregation of basal fate determinants Pon/Numb in Drosophila neuroblasts [53]. Theoretical work suggests a unique feature of LLPS in cell polarity may be its ability to lock in a polar distribution of phase separated material following a transient stimulus without additional energy input [54].

When taken to the extreme, this paradigm of local switching between rapid and slow diffusing states morphs into a simple diffusion and capture model in which an immobile scaffold binds to and thereby locally traps an otherwise randomly moving species (Figure 2D). Such a mechanism guides anterior and posterior trapping of the anterior marker Bicoid and the posterior marker Oskar, downstream of PAR polarization of the Drosophila oocyte, allowing randomly or weakly oriented transport to be converted to stable asymmetry [55,56]. Thus, consideration of the mobilities of molecules and their spatial regulation supports a generic mechanism for the polarization of molecules in cells.

\section{Regulation of polarity in space and time}

The ability of polarity complexes to switch between states provides cells with flexible and highly controllable systems for regulating the polarization and downstream outputs of polarity networks in space and time. This is particularly critical as conserved polarity molecules are often reused and reconfigured as development proceeds. Even within the C. elegans embryo, the PAR polarity machinery is deployed across multiple contexts, first to drive cell-autonomous symmetry-breaking and anterior-posterior axis specification in the zygote, then to specify contact dependent radial polarity in early blastomeres, and subsequently to specify apical-basal polarity in a variety of epithelial tissues [57]. In each case, the configuration of polarity proteins and the symmetrybreaking signals to which the PAR network respond vary. Thus, spatiotemporal control over polarity networks themselves is central to their proper function.

\section{Developmental switches}

Developmentally regulated changes in PAR polarity are often associated with cell type specific switches in the composition of PAR complexes and changing demands on their functions in cells. We have already seen how directionality of Par3 transport on microtubules is subject to cell-type 
specific regulation. In Drosophila, sensory organ precursors (SOP) derive from PAR-polarized epithelia. Before entry in mitosis, Par3 (Baz in fly) which is normally positioned uniformly at the apical junctions in the epithelia, becomes planar polarized thanks to its interaction with Meru, a SOP-specific planar cell polarity (PCP) adaptor [58]. Baz therefore shifts from responding to apical basal polarity (ABP) components to PCP modules to polarize the SOP cell along the PCP axis prior to cell division (Figure $3 A$ ). Interestingly such a functional switch between ABP and PCP also occurs in vertebrates, although the molecular details differ [59](Figure 3A). In developing epithelia, Baz again is subject to regulation of its localization and function, here through its phosphorylation by aPKC, which alters the balance of Par-containing functional complexes. Baz initially localizes apically to direct recruitment of Par6, aPKC and other apical components [6,60]. Subsequently, phosphorylation by aPKC triggers the release of Baz from these apical complexes and its relocation to the apical junctions; while Par6/aPKC associates with Crumbs to help specify apical membrane identity in a Cdc42-dependent fashion [61,62] (Figure 3B). In yet another example, the polarity component Yurt (Lulu in mammals) undergoes a developmental switch in localization. Early on, Yurt plays a role in preventing invasion of apical components such as Crumbs and aPKC into basolateral regions. Yurt activity relies on its oligomerized state and is restricted basolaterally by negative aPKC phosphorylation. Epithelium maturation leads to Yurt oligomers resisting aPKC phosphorylation, thus extending apically to suppress local function of Crumbs, and thereby limiting apical domain size [5,63](Figure 3B).

\section{Cell cycle control}

The cell cycle places constraints on the function of PAR polarity-related protein complexes. Reviewed extensively elsewhere in this issue, mitosis poses unique challenges for polarized cells due to the need to coordinate cell polarity with the dramatic spatial re-organization cell division entails. We already saw a glimpse of this in the regulation of asymmetric division of epithelial SOP cells. In fact, even in symmetrically-dividing epithelia, polarity regulators play important roles, often associated with control of the mitotic spindle alignment. In this case they ensure division occurs parallel to the plane of the epithelial tissue to maintain tissue architecture. In Drosophila follicular epithelia, spindle alignment requires remodelling of the Dlg/Scrib/Lgl complex. In nonmitotic epithelia, the Dlg/Scrib/Lgl complex is implicated in apical basal polarity [64]. However, during mitosis of various epithelial cells, Dlg/Scrib become important for proper spindle alignment in the plane of the epithelia $[65,66]$. New work suggests that at least in Drosophila follicular 
epithelia, this mitotic shift in function requires phosphorylation of Lgl by the cell cycle kinase Aurora A, which releases $\mathrm{Lgl}$ from the Dlg/Scrib complex [67,68]. As $\mathrm{Lgl}$ and the spindle orientation factor Pins are bound by the same phosphoserine binding region within the guanylate cyclase domain of Dlg [69,70], phosphorylation of Lgl by Aurora A could allow Dlg to associate with Pins to orient the spindle in these cells. Following division, activity of the PP1 phosphatase allows Lgl back onto the membrane to act in concert with Scrib in maintaining basolateral membrane identity [71]. Finally, recent data suggest that Aurora and Polo kinases can tune activity of PAR complex proteins. In Drosophila neural precursors, Aurora A modulates PAR complex composition by phosphorylation of PAR-6 to activate aPKC [72], while in the $C$. elegans zygote, Aurora A and the Polo kinase PLK-1 control the levels and oligomeric state of PAR-3 to ensure proper symmetry-breaking in response to spatial cues $[24,73]$.

\section{Outlook}

Far from being stable complexes, recent work has revealed the dynamic behaviour of polarity proteins such as PARs, to be integral in their function. This capacity for dynamic remodelling also favours their ability to segregate in space, and to adapt to changing contexts, such as during tissue development, within the cell cycle, or in different cell types. We are only beginning to understand the complex regulation of these dynamic behaviours.

\section{Acknowledgements}

The authors acknowledge support from the Francis Crick Institute (NWG), which receives its core funding from Cancer Research UK (FC001086), the UK Medical Research Council (FC001086), and the Wellcome Trust (FC001086), and the Fondation ARC pour la recherche sur le cancer (FP).

\section{Disclosure}

The authors declare no conflict of interest. 


\section{References}

1. Goldstein B, Macara IG: The PAR proteins: fundamental players in animal cell polarization. Dev Cell 2007, 13:609-622.

2. McCaffrey LM, Macara IG: Signaling pathways in cell polarity. Cold Spring Harb Perspect Biol 2012, 4.

3. Goehring NW: PAR polarity: from complexity to design principles. Exp Cell Res 2014, 328:258-266.

4. Morais-de-Sa E, Mukherjee A, Lowe N, St Johnston D: SImb antagonises the aPKC/Par-6 complex to control oocyte and epithelial polarity. Development 2014, 141:29842992.

5. Laprise P, Beronja S, Silva-Gagliardi NF, Pellikka M, Jensen AM, McGlade CJ, Tepass U: The FERM protein Yurt is a negative regulatory component of the Crumbs complex that controls epithelial polarity and apical membrane size. Dev Cell 2006, 11:363-374.

6. Tepass U: The apical polarity protein network in Drosophila epithelial cells: regulation of polarity, junctions, morphogenesis, cell growth, and survival. Annu Rev Cell Dev Biol 2012, 28:655-685.

7. Rodriguez-Boulan E, Macara IG: Organization and execution of the epithelial polarity programme. Nat Rev Mol Cell Biol 2014, 15:225-242.

8. Flores-Benitez D, Knust E: Dynamics of epithelial cell polarity in Drosophila: how to regulate the regulators? Curr Opin Cell Biol 2016, 42:13-21.

9. Zimyanin VL, Belaya K, Pecreaux J, Gilchrist MJ, Clark A, Davis I, St Johnston D: In vivo imaging of oskar mRNA transport reveals the mechanism of posterior localization. Cell 2008, 134:843-853.

10. Brendza RP, Serbus LR, Duffy JB, Saxton WM: A function for kinesin I in the posterior transport of oskar mRNA and Staufen protein. Science 2000, 289:2120-2122.

11. Kim DJ, Martinez-Lemus LA, Davis GE: EB1, p150Glued, and Clasp1 control endothelial tubulogenesis through microtubule assembly, acetylation, and apical polarization. Blood 2013, 121:3521-3530.

12. Kamei M, Saunders WB, Bayless KJ, Dye L, Davis GE, Weinstein BM: Endothelial tubes assemble from intracellular vacuoles in vivo. Nature 2006, 442:453-456.

13. Harris TJ, Peifer M: The positioning and segregation of apical cues during epithelial polarity establishment in Drosophila. J Cell Biol 2005, 170:813-823.

14. Nishimura T, Kato K, Yamaguchi T, Fukata Y, Ohno S, Kaibuchi K: Role of the PAR-3-KIF3 complex in the establishment of neuronal polarity. Nat Cell Biol 2004, 6:328-334.

15. Schmoranzer J, Fawcett JP, Segura M, Tan S, Vallee RB, Pawson T, Gundersen GG: Par3 and dynein associate to regulate local microtubule dynamics and centrosome orientation during migration. Curr Biol 2009, 19:1065-1074.

16. Jouette J, Guichet A, Claret SB: Dynein-mediated transport and membrane trafficking control PAR3 polarised distribution. Elife 2019, 8.

* During Drosophila oogenesis, PAR proteins distribute into two opposite domains to drive the polarization of downstream cell fate determinants. This work reveals new molecular mechanism into Par3 exclusion from posterior domains and its transport to the anterior membranes. 
17. Leduc C, Etienne-Manneville S: Regulation of microtubule-associated motors drives intermediate filament network polarization. J Cell Biol 2017, 216:1689-1703.

* This study elegantly demonstrates that leading-edge enrichment of active aPKC/Cdc42 proteins induces a switch in motor dependent transport of intermediate filaments (IF), from a dual direction mode using both dynein and kinesin, to a kinesin-biased mode to promote directional IF transport towards the leading edge.

18. Watanabe N, Mitchison TJ: Single-molecule speckle analysis of actin filament turnover in lamellipodia. Science 2002, 295:1083-1086.

19. Lin $\mathrm{CH}$, Espreafico EM, Mooseker MS, Forscher P: Myosin drives retrograde F-actin flow in neuronal growth cones. Neuron 1996, 16:769-782.

20. Forscher P, Smith SJ: Actions of cytochalasins on the organization of actin filaments and microtubules in a neuronal growth cone. J Cell Biol 1988, 107:1505-1516.

21. Maiuri P, Rupprecht JF, Wieser S, Ruprecht V, Benichou O, Carpi N, Coppey M, De Beco S, Gov N, Heisenberg CP, et al.: Actin flows mediate a universal coupling between cell speed and cell persistence. Cell 2015, 161:374-386.

22. Munro E, Nance J, Priess JR: Cortical flows powered by asymmetrical contraction transport PAR proteins to establish and maintain anterior-posterior polarity in the early C. elegans embryo. Dev Cell 2004, 7:413-424.

23. Goehring NW, Trong PK, Bois JS, Chowdhury D, Nicola EM, Hyman AA, Grill SW: Polarization of PAR proteins by advective triggering of a pattern-forming system. Science 2011, 334:1137-1141.

24. Dickinson DJ, Schwager F, Pintard L, Gotta M, Goldstein B: A Single-Cell Biochemistry Approach Reveals PAR Complex Dynamics during Cell Polarization. Dev Cell 2017, 42:416-434 e411.

** This study, along with 25-26, reveal that association of Par6/aPKC clustered PAR-3 is required for efficient polarization of the $\mathrm{C}$. elegans zygote by actomyosin cortical flows. This study makes use of a novel single molecule technique using single embryo lystates to show how Par3 clustering is regulated by the cell cycle kinase PLK-1 to maximize cluster size during the polarization phase.

25. Rodriguez J, Peglion F, Martin J, Hubatsch L, Reich J, Hirani N, Gubieda AG, Roffey J, Fernandes AR, St Johnston D, et al.: aPKC Cycles between Functionally Distinct PAR Protein Assemblies to Drive Cell Polarity. Dev Cell 2017, 42:400-415 e409.

** This study demonstrates the ability of aPKC to cycle between distinct functional states, one associated with PAR-3 that is required for sensing asymmetric cues, and another CDC-42-associated state required for aPKC activity and its ability to exclude opposing PAR species from the anterior domain.

26. Wang SC, Low TYF, Nishimura Y, Gole L, Yu W, Motegi F: Cortical forces and CDC-42 control clustering of PAR proteins for Caenorhabditis elegans embryonic polarization. Nat Cell Biol 2017, 19:988-995.

* This study provides evidence that the clustered state of PAR3 is also regulated by contractility of the actomyosin cortex, providing a link between actomyosin cortical activity and Par3 clustering. 
27. Goehring NW, Hoege C, Grill SW, Hyman AA: PAR proteins diffuse freely across the anterior-posterior boundary in polarized C. elegans embryos. J Cell Biol 2011, 193:583-594.

28. Sailer A, Anneken A, Li Y, Lee S, Munro E: Dynamic Opposition of Clustered Proteins Stabilizes Cortical Polarity in the C. elegans Zygote. Dev Cell 2015, 35:131-142.

29. Robin FB, McFadden WM, Yao B, Munro EM: Single-molecule analysis of cell surface dynamics in Caenorhabditis elegans embryos. Nat Methods 2014, 11:677-682.

30. Arata Y, Hiroshima M, Pack CG, Ramanujam R, Motegi F, Nakazato K, Shindo Y, Wiseman PW, Sawa H, Kobayashi TJ, et al.: Cortical Polarity of the RING Protein PAR-2 Is Maintained by Exchange Rate Kinetics at the Cortical-Cytoplasmic Boundary. Cell Rep 2016, 16:2156-2168.

31. Ramanujam R, Han Z, Zhang Z, Kanchanawong P, Motegi F: Establishment of the PAR-1 cortical gradient by the aPKC-PRBH circuit. Nat Chem Biol 2018, 14:917-927.

32. Motegi F, Zonies S, Hao Y, Cuenca AA, Griffin E, Seydoux G: Microtubules induce selforganization of polarized PAR domains in Caenorhabditis elegans zygotes. Nat Cell Biol 2011, 13:1361-1367.

33. Moravcevic K, Mendrola JM, Schmitz KR, Wang YH, Slochower D, Janmey PA, Lemmon MA: Kinase associated-1 domains drive MARK/PAR1 kinases to membrane targets by binding acidic phospholipids. Cell 2010, 143:966-977.

34. Bailey MJ, Prehoda KE: Establishment of Par-Polarized Cortical Domains via Phosphoregulated Membrane Motifs. Dev Cell 2015, 35:199-210.

35. Dong W, Zhang X, Liu W, Chen YJ, Huang J, Austin E, Celotto AM, Jiang WZ, Palladino MJ, Jiang $Y$, et al.: A conserved polybasic domain mediates plasma membrane targeting of $\mathrm{Lgl}$ and its regulation by hypoxia. J Cell Biol 2015, 211:273-286.

36. Smith CA, Lau KM, Rahmani Z, Dho SE, Brothers G, She YM, Berry DM, Bonneil E, Thibault $P$, Schweisguth $F$, et al.: aPKC-mediated phosphorylation regulates asymmetric membrane localization of the cell fate determinant Numb. EMBO $J$ 2007, 26:468-480.

37. Hannaford MR, Ramat A, Loyer N, Januschke J: aPKC-mediated displacement and actomyosin-mediated retention polarize Miranda in Drosophila neuroblasts. Elife 2018, 7.

38. Harris TJC: Protein clustering for cell polarity: Par-3 as a paradigm. F1000Res 2017, 6:1620.

39. Kono KY, S;Fujita, I; Okada,Y; Shitamukai,A; Shibata, T; Matsuzaki, F: Reconstruction of Par polarity in apolar cells reveals a dynamic process of cortical polarization bioRxiv 2019.

40. Katz ZB, English BP, Lionnet T, Yoon YJ, Monnier N, Ovryn B, Bathe M, Singer RH: Mapping translation 'hot-spots' in live cells by tracking single molecules of mRNA and ribosomes. Elife 2016, 5.

41. Yan X, Hoek TA, Vale RD, Tanenbaum ME: Dynamics of Translation of Single mRNA Molecules In Vivo. Cell 2016, 165:976-989.

42. Swaminathan $R$, Hoang $C P$, Verkman AS: Photobleaching recovery and anisotropy decay of green fluorescent protein GFP-S65T in solution and cells: cytoplasmic viscosity probed by green fluorescent protein translational and rotational diffusion. Biophys J 1997, 72:1900-1907.

43. Daniels BR, Perkins EM, Dobrowsky TM, Sun SX, Wirtz D: Asymmetric enrichment of PIE-1 in the Caenorhabditis elegans zygote mediated by binary counterdiffusion. $J$ Cell Biol 2009, 184:473-479. 
44. Daniels BR, Dobrowsky TM, Perkins EM, Sun SX, Wirtz D: MEX-5 enrichment in the C. elegans early embryo mediated by differential diffusion. Development 2010, 137:2579-2585.

45. Griffin EE, Odde DJ, Seydoux G: Regulation of the MEX-5 gradient by a spatially segregated kinase/phosphatase cycle. Cell 2011, 146:955-968.

46. Wu Y, Han B, Li Y, Munro E, Odde DJ, Griffin EE: Rapid diffusion-state switching underlies stable cytoplasmic gradients in the Caenorhabditis elegans zygote. Proc Natl Acad Sci U S A 2018, 115:E8440-E8449.

* $\quad$ Combining modeling, genetics, and single particle tracking, this study highlights how spatially-regulated switching between fast and slow-diffusing states allows stable cytoplasmic gradient formation in the $C$. elegans zygote.

47. Wu Y, Zhang H, Griffin EE: Coupling between cytoplasmic concentration gradients through local control of protein mobility in the Caenorhabditis elegans zygote. $\mathrm{Mol}$ Biol Cell 2015, 26:2963-2970.

48. Han B, Antkowiak KR, Fan X, Rutigliano M, Ryder SP, Griffin EE: Polo-like Kinase Couples Cytoplasmic Protein Gradients in the C. elegans Zygote. Curr Biol 2018, 28:60-69 e68.

49. Wu Y HB, Smith J, Singh A, Griffin EE Single molecule dynamics of the P granule scaffold MEG-3 in the C. elegans zygote. Molecular Biology of the Cell 2019, 30:333345.

50. Brangwynne CP, Eckmann CR, Courson DS, Rybarska A, Hoege C, Gharakhani J, Julicher F, Hyman AA: Germline $\mathbf{P}$ granules are liquid droplets that localize by controlled dissolution/condensation. Science 2009, 324:1729-1732.

51. Smith J, Calidas D, Schmidt H, Lu T, Rasoloson D, Seydoux G: Spatial patterning of $\mathbf{P}$ granules by RNA-induced phase separation of the intrinsically-disordered protein MEG-3. Elife 2016, 5.

52. Saha S, Weber CA, Nousch M, Adame-Arana O, Hoege C, Hein MY, Osborne-Nishimura E, Mahamid J, Jahnel M, Jawerth L, et al.: Polar Positioning of Phase-Separated Liquid Compartments in Cells Regulated by an mRNA Competition Mechanism. Cell 2016, 166:1572-1584 e1516.

53. Shan Z, Tu Y, Yang Y, Liu Z, Zeng M, Xu H, Long J, Zhang M, Cai Y, Wen W: Basal condensation of Numb and Pon complex via phase transition during Drosophila neuroblast asymmetric division. Nat Commun 2018, 9:737.

* $\quad$ Coupling structural biochemistry with in vivo data, this study highlights the role of phase separation in promoting the asymetric enrichment of Pon and Numb during Drosophila neuroblast asymmetric division.

54. Dine E, Gil AA, Uribe G, Brangwynne CP, Toettcher JE: Protein Phase Separation Provides Long-Term Memory of Transient Spatial Stimuli. Cell Syst 2018, 6:655-663 e655.

* In a proof of principle, this study highlights how a local phase separation in response to a stimulus can be kinetically trapped to provide spatial memory that persists long after the stimulus is withdrawn. 
55. Trovisco V, Belaya K, Nashchekin D, Irion U, Sirinakis G, Butler R, Lee JJ, Gavis ER, St Johnston D: bicoid mRNA localises to the Drosophila oocyte anterior by random Dynein-mediated transport and anchoring. Elife 2016, 5.

56. Lu W, Lakonishok M, Serpinskaya AS, Kirchenbuechler D, Ling SC, Gelfand VI: Ooplasmic flow cooperates with transport and anchorage in Drosophila oocyte posterior determination. J Cell Biol 2018, 217:3497-3511.

** By decoupling kinesin-1 requirements in generating the ooplasmic flow and transport on MTs tracks, this work teases apart their respective roles in oskar mRNA asymmetry during Drosophila oogenesis, revealing partial redundancy. They further show that the myosin MyoV functions as a membrane anchor to trap the RNP particles when they reach the cortex.

57. Nance J: Getting to know your neighbor: cell polarization in early embryos. J Cell Biol 2014, 206:823-832.

58. Banerjee JJ, Aerne BL, Holder MV, Hauri S, Gstaiger M, Tapon N: Meru couples planar cell polarity with apical-basal polarity during asymmetric cell division. Elife 2017, 6.

* Identification of Meru, the fly RASSF homolog, as an adaptor protein mediating the apico-basal to planar cell polarity switch of Par3 in Drosophila sensory organ precursor cells.

59. Chuykin I, Ossipova O, Sokol SY: Par3 interacts with Prickle3 to generate apical PCP complexes in the vertebrate neural plate. Elife 2018, 7.

60. Ohno S GS, and Hirose T (Ed): Chapter 1: The PAR3-aPKC-PAR6 complex: Springer; 2015.

61. Morais-de-Sa E, Mirouse V, St Johnston D: aPKC phosphorylation of Bazooka defines the apical/lateral border in Drosophila epithelial cells. Cell 2010, 141:509-523.

62. Walther RF, Pichaud F: Crumbs/DaPKC-dependent apical exclusion of Bazooka promotes photoreceptor polarity remodeling. Curr Biol 2010, 20:1065-1074.

63. Gamblin CL, Parent-Prevost F, Jacquet K, Biehler C, Jette A, Laprise P: Oligomerization of the FERM-FA protein Yurt controls epithelial cell polarity. J Cell Biol 2018, 217:3853-3862.

* This study reveals the molecular mechanism underlying aPKC antagonism against the FERM-FA domain protein Yurt and its mammalian homolog Lulu. They identify residues in the FA motif responsible for Yrt oligomerization which favours its antagonistic interaction with Crumbs. aPKC impacts Yrt by phosphorylating the FA domain and preventing its oligomerization state.

64. Yamanaka T, Ohno S: Role of Lgl/Dlg/Scribble in the regulation of epithelial junction, polarity and growth. Front Biosci 2008, 13:6693-6707.

65. Bergstralh DT, Lovegrove HE, St Johnston D: Discs large links spindle orientation to apical-basal polarity in Drosophila epithelia. Curr Biol 2013, 23:1707-1712.

66. Nakajima Y, Meyer EJ, Kroesen A, McKinney SA, Gibson MC: Epithelial junctions maintain tissue architecture by directing planar spindle orientation. Nature 2013, 500:359-362. 
67. Bell GP, Fletcher GC, Brain R, Thompson BJ: Aurora kinases phosphorylate Lgl to induce mitotic spindle orientation in Drosophila epithelia. Curr Biol 2015, 25:61-68.

68. Carvalho CA, Moreira S, Ventura G, Sunkel CE, Morais-de-Sa E: Aurora A triggers Lgl cortical release during symmetric division to control planar spindle orientation. Curr Biol 2015, 25:53-60.

69. Johnston CA, Hirono K, Prehoda KE, Doe CQ: Identification of an AuroraA/PinsLINKER/DIg spindle orientation pathway using induced cell polarity in $\mathbf{S 2}$ cells. Cell 2009, 138:1150-1163.

70. Zhu J, Shang Y, Wan Q, Xia Y, Chen J, Du Q, Zhang M: Phosphorylation-dependent interaction between tumor suppressors Dlg and Lgl. Cell Res 2014, 24:451-463.

71. Moreira S, Osswald M, Ventura G, Goncalves M, Sunkel CE, Morais-de-Sa E: PP1Mediated Dephosphorylation of Lgl Controls Apical-basal Polarity. Cell Rep 2019, 26:293-301 e297.

72. Wirtz-Peitz F, Nishimura T, Knoblich JA: Linking cell cycle to asymmetric division: Aurora-A phosphorylates the Par complex to regulate Numb localization. Cell 2008, 135:161-173.

73. Reich JD, Hubatsch L, Illukkumbura R, Peglion F, Bland T, Hirani N, Goehring NW: Regulated activation of the PAR polarity network ensures a timely and specific response to spatial cues. Current Biology 2019 (in press).

74. Benton R, St Johnston D: A conserved oligomerization domain in drosophila Bazooka/PAR-3 is important for apical localization and epithelial polarity. Curr Biol 2003, 13:1330-1334.

75. Feng W, Wu H, Chan LN, Zhang M: The Par-3 NTD adopts a PB1-like structure required for Par-3 oligomerization and membrane localization. EMBO J 2007, 26:2786-2796.

76. Mizuno K, Suzuki A, Hirose T, Kitamura K, Kutsuzawa K, Futaki M, Amano Y, Ohno S: Selfassociation of PAR-3-mediated by the conserved $\mathrm{N}$-terminal domain contributes to the development of epithelial tight junctions. J Biol Chem 2003, 278:31240-31250.

77. Strand D, Jakobs R, Merdes G, Neumann B, Kalmes A, Heid HW, Husmann I, Mechler BM: The Drosophila lethal(2)giant larvae tumor suppressor protein forms homooligomers and is associated with nonmuscle myosin II heavy chain. J Cell Biol 1994, 127:1361-1373.

78. Meca J, Massoni-Laporte A, Martinez D, Sartorel E, Loquet A, Habenstein B, McCusker D: Avidity-driven polarity establishment via multivalent lipid-GTPase module interactions. EMBO J 2018.

79. Benton R, St Johnston D: Drosophila PAR-1 and 14-3-3 inhibit Bazooka/PAR-3 to establish complementary cortical domains in polarized cells. Cell 2003, 115:691704.

80. Nomanbhoy TK, Erickson JW, Cerione RA: Kinetics of Cdc42 membrane extraction by Rho-GDI monitored by real-time fluorescence resonance energy transfer. Biochemistry 1999, 38:1744-1750.

81. Carol RJ, Takeda S, Linstead P, Durrant MC, Kakesova H, Derbyshire P, Drea S, Zarsky V, Dolan L: A RhoGDP dissociation inhibitor spatially regulates growth in root hair cells. Nature 2005, 438:1013-1016.

82. Hwang JU, Wu G, Yan A, Lee YJ, Grierson CS, Yang Z: Pollen-tube tip growth requires a balance of lateral propagation and global inhibition of Rho-family GTPase activity. J Cell Sci 2010, 123:340-350.

83. Kistler KE, Trcek T, Hurd TR, Chen R, Liang FX, Sall J, Kato M, Lehmann R: Phase transitioned nuclear Oskar promotes cell division of Drosophila primordial germ cells. Elife 2018, 7. 
84. Vanzo NF, Ephrussi A: Oskar anchoring restricts pole plasm formation to the posterior of the Drosophila oocyte. Development 2002, 129:3705-3714.

85. Forrest KM, Gavis ER: Live imaging of endogenous RNA reveals a diffusion and entrapment mechanism for nanos mRNA localization in Drosophila. Curr Biol 2003, 13:1159-1168. 


\section{Figure 1: Generating asymmetry through directed transport}

(a, b) Examples of directional transport driven by polarized cytoskeletal tracks and preferential cargo-motor association. (a) In the oocyte, an asymmetric MT network drives polarized transport of oskar and bicoid mRNA as well as PAR proteins. Their differential accumulation arises from preferential association of cargoes with distinct motors: dynein drives accumulation of bicoid and Bazooka to the anterior, while kinesin drives accumulation of oskar to the posterior. (b) A PAR-dependent switch in motor affinity allows polarized accumulation of intermediate filaments (IF) during cell migration. (c) Polarized transport by bulk cortical flow (advection). At left, cortical actomyosin flow in the $C$. elegans zygote preferentially transports a pool of PAR-6/aPKC that is associated with oligomers of PAR-3 to the anterior. The oligomeric state of PAR-3 is tightly regulated by cell-cycle kinase PLK-1 and cortex contractility itself and is essential for its transport. At right - direct association with the actin cortex allows polarity cues to be advected by retrograde actin flow during cell migration. The binding-affinity of molecules for F-actin shapes their concentration gradients, reinforcing the polarity of the moving cell. 


\section{Figure 2: Asymmetry by diffusion-based local retention mechanisms}

Asymmetric switching between slow- and fast-moving states allow for polarized accumulation.

(a) Interaction with the plasma membrane lipid bilayer restricts diffusion. Membrane association is often associated with oligomerization or complex formation providing multivalent interactions that enable stable membrane interaction and activity. Displacement from the membrane shifts molecules to a fast diffusing cytoplasmic state. This switch may be triggered by phosphorylation on basic hydrophobic residues or oligomerization domains important for membrane binding, or, in the case of RhoGTPases, by their extraction via RhoGDI proteins. See [24, 30-32, 34, 35, 37, 38, 63, 74-82]. (b) Cytoplasmic fate determinants can be slowed by switching into an RNAbound state. By spatially regulating interconversion between an RNA-trapped state and an unbound, fast diffusing state, molecules can be segregated asymmetrically in the cytoplasm. See [43, 45-49]. (c) Liquid-Liquid phase separation (LLPS) also allows for local retention as phase separated droplets diffuse very slowly. Spatial regulation of a molecule's ability to partition into droplets allows them to be retained asymmetrically. See [50, 51, 53, 83]. (d) Capture of randomly diffusing molecules by immobile anchors represents an extreme case of diffusion-based retention mechanism. See [55, 56, 84, 85]. 


\section{Figure 3: Functional switching of polarity modules during tissue development}

(a) Context-specific switching of Par3 polarization from apical-basal to planar polarized during development. Switching is achieved by developmentally regulated expression of the polarity adaptors Meru in sensory organ precursor cells (Drosophila) and Prickle3 in vertebrate neuroepithelium, which link Par3 to components of the planar cell polarity pathways. (b) Temporal switches in polarity protein behaviour during development of apical-basal polarized epithelia. At left, Par3 (orange) is shown initially enriched at nascent cell-cell contacts $(\mathrm{J})$ and recruits aPKC/Par6 apically (A). Par3 is then phosphorylated by aPKC, presumably upon activation by apical enrichment of $\mathrm{Cdc} 42$, which triggers its exclusion from apical membrane and relocation to the junctions. In parallel aPKC/Par6 is released from Par3 and retained apically via interaction with the Crumbs complex. At right, Yurt (Lulu in mammals, purple) extends apically during epithelium maturation. Yurt inhibition of Crumbs depends on its oligomerized state (oligo). This state is suppressed by aPKC, which promotes the monomeric state (mono), restricting Yurt activity to the basolateral domain (BL). As the epithelium develops, phosphorylation of Yurt is reduced, possibly due to increased phosphatase activity or insensitivity to aPKC, promoting its oligomerization, which helps restrict the size of the Crumbsenriched apical domain. 


\section{(a)}

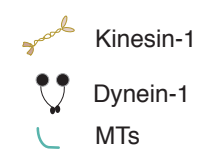

biased polarity of MTs network

1. Baz (Par3)

- oskar mRNA

- bicoid mRNA (c)

Migrating cell

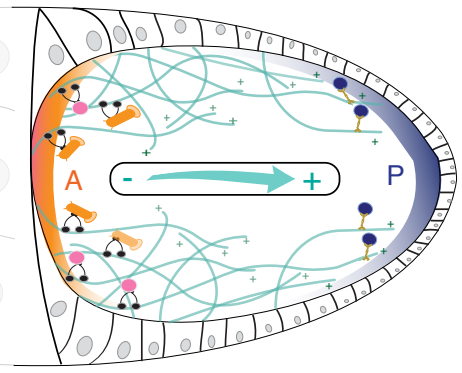

retrograde flow
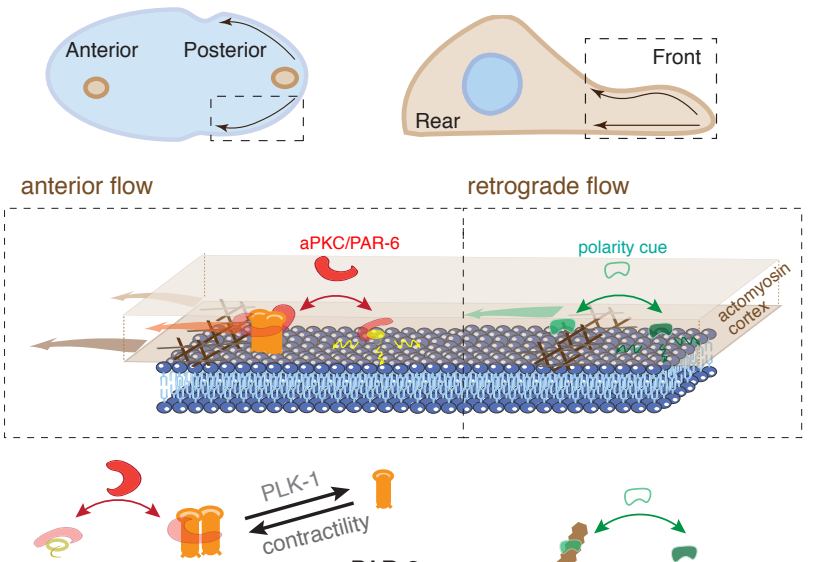

PAR-3

CDC-42 state PAR-3 state monomeric (diffuse) LEront-enriched Cdc42/aPKC $\uparrow$ Polarized migrating cell

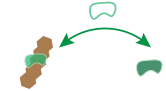

Actin-bound Actin-Free (advected) (Diffuse) (diffuse)

(advected)

Dynein-bound

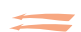

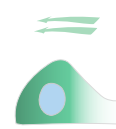

(b)

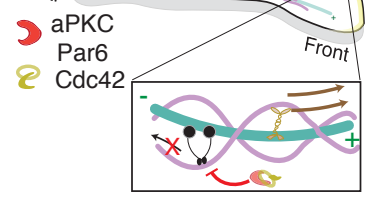




\begin{tabular}{|c|c|c|c|}
\hline$\underset{(\text { Fast })}{\text { State } 1}$ & $\begin{array}{c}\text { State } 2 \\
\text { (Slow) }\end{array}$ & Molecular Mechanisms & Examples \\
\hline (a) Membrane Interaction & $\begin{array}{l}\text { Membrane } \\
\text { bound }\end{array}$ & $\begin{array}{l}\text { Homo-oligomerization } \\
\text { Hetero-oligomerization } \\
\text { aPKC-dependent phosphorylation } \\
\text { Par1-dependent phosphorylation } \\
\text { PLK-1-dependent phosphorylation } \\
\text { RhoGDI-dependent membrane extraction }\end{array}$ & $\begin{array}{l}\text { Par3 [38,74-76] } \\
\text { PAR-2 [30] } \\
\text { Lgl [77] } \\
\text { Yurt [63] } \\
\text { PAR-1/PAR-2 [31] } \\
\text { aPKC/Par6/Par3 [24] } \\
\text { Cdc42/Bem1/Cdc24 [78] } \\
\text { PAR-1 [31] } \\
\text { PAR-2 [32] } \\
\text { Lgl [34,35] } \\
\text { Miranda, Numb [34,37] } \\
\text { Par3 [32,79] } \\
\text { Par3 [24] } \\
\text { Cdc42 [80] } \\
\text { ROPs (root hair [81], pollen tube [82]) }\end{array}$ \\
\hline (b) Cytoplasn & Trapped & $\begin{array}{l}\text { RNP trapping } \\
\text { PP2A-dependent binding } \\
\text { PAR-1-dependent unbinding } \\
\text { MEX-5 / PLK-1-dependent unbinding }\end{array}$ & $\begin{array}{l}\text { MEX-5 [45] } \\
\text { PIE-1 [43], POS-1, MEX-1 [46-48] } \\
\text { MEG-3[49] } \\
\text { MEX-5 [45] } \\
\text { PIE-1, MEX-1, POS-1 [48] }\end{array}$ \\
\hline (c) Liquid-Liqu & Condensed & $\begin{array}{l}\text { RNA-protein interactions } \\
\text { MEX-5 dependent } \\
\text { Oskar dependent } \\
\text { Protein-protein interactions }\end{array}$ & $\begin{array}{l}\text { P-granules: } \\
\text { PGL-1/GLH-1 [50], MEG-3 [51] } \\
\text { Nuclear granules: } \\
\text { Oskar/Vasa [83] } \\
\text { Numb/Pon [53] }\end{array}$ \\
\hline (d) Diffusion a & Captured & $\begin{array}{l}\text { Myosin-dependent anchor } \\
\text { Oskar-dependent anchor } \\
\text { Unknown anchor }\end{array}$ & $\begin{array}{l}\text { oskar mRNA/ Staufen [56] } \\
\text { Germ Granules [84] } \\
\text { nanos mRNA [85] } \\
\text { bicoid mRNA [55] }\end{array}$ \\
\hline
\end{tabular}


(a)

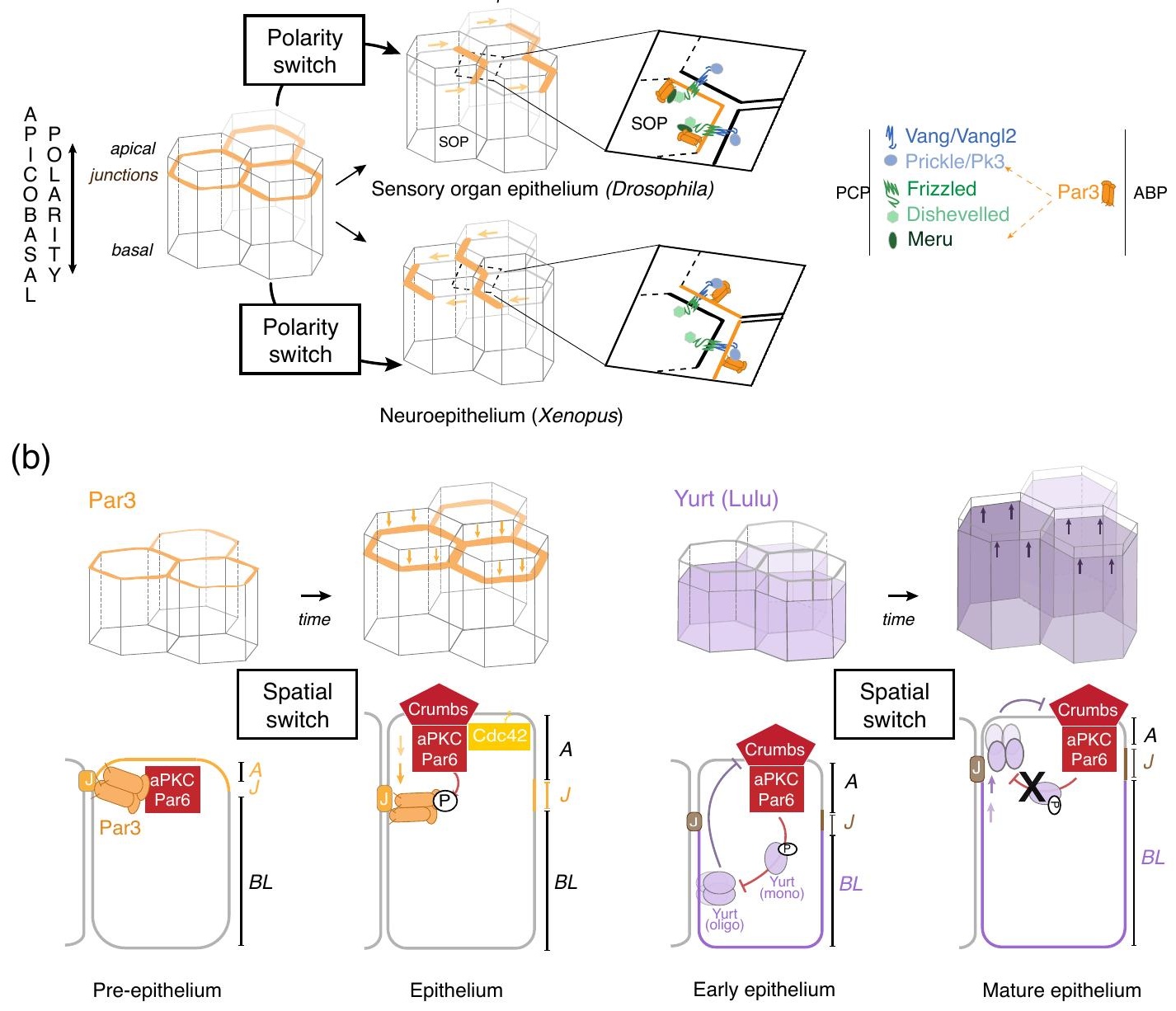

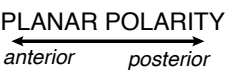

y. Vang/Vangl2

- Prickle/Pk3.

Frizzled

Dishevelled

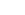

B R

$S T$

A $Y$

Mature epithelium 